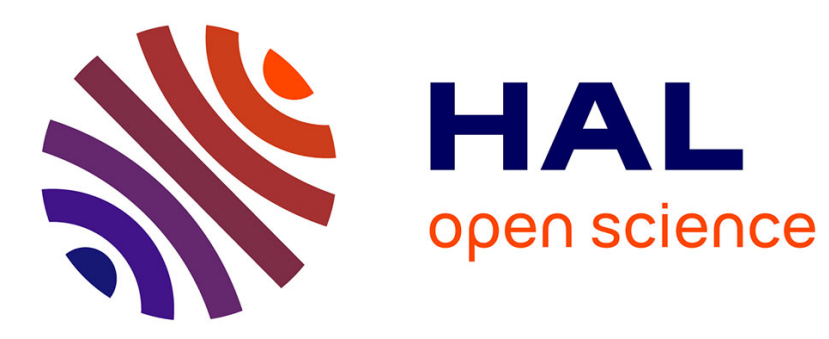

\title{
Cascade of Nonlinear Entropy and Statistics to Discriminate Fetal Heart Rates
}

Amira J. Zaylaa, Soha Saleh, Fadi N. Karameh, Ziad Nahas, Ayache Bouakaz

\section{To cite this version:}

Amira J. Zaylaa, Soha Saleh, Fadi N. Karameh, Ziad Nahas, Ayache Bouakaz. Cascade of Nonlinear Entropy and Statistics to Discriminate Fetal Heart Rates. 2016 3rd International Conference on Advances in Computational Tools for Engineering Applications (ACTEA), Jul 2016, Notre Dame University, France. inserm-01337555

\section{HAL Id: inserm-01337555 https://www.hal.inserm.fr/inserm-01337555}

Submitted on 27 Jun 2016

HAL is a multi-disciplinary open access archive for the deposit and dissemination of scientific research documents, whether they are published or not. The documents may come from teaching and research institutions in France or abroad, or from public or private research centers.
L'archive ouverte pluridisciplinaire HAL, est destinée au dépôt et à la diffusion de documents scientifiques de niveau recherche, publiés ou non, émanant des établissements d'enseignement et de recherche français ou étrangers, des laboratoires publics ou privés. 


\title{
Cascade of Nonlinear Entropy and Statistics to Discriminate Fetal Heart Rates
}

\author{
A. J. Zaylaa ${ }^{1,2}$, S. Saleh ${ }^{3}$, F. N. Karameh ${ }^{1}$, Z. Nahas ${ }^{1}$ and A. Bouakaz ${ }^{4}$ \\ 1 American University of Beirut and Medical Center, Lebanon \\ az56@aub.edu.lb, fk14@aub.edu.lb, zn17@aub.edu.lb \\ ${ }^{2}$ Research Platform in Environmental and Health Sciences, \\ Doctoral School of Science and Technology, Lebanese University, Beirut, Lebanon \\ ${ }^{3}$ Kessler Institute for Rehabilitation, Kessler Foundation, USA. ssaleh@kesslerfoundation.org \\ ${ }^{4}$ CHRU Bretonneau Hospital, François-Rabelais University of Tours, France. bouakaz@med.univ-tours.fr
}

\begin{abstract}
Fetal heart rate discrimination is an evolving field in biomedical engineering with many efforts dedicated to avoid preterm deliveries by way of improving fetus monitoring methods and devices. Entropy analysis is a nonlinear signal analysis technique that has been progressively developed to improve the discriminability of a several physiological signals, with Kernel based entropy parameters (KBEPs) found advantageous over standard techniques. This study is the first to apply KBEPs to analyze fetal heart rates. Specifically, it explores the usability of the cutting-edge nonlinear KBEPs in discriminating between healthy fetuses and fetuses under distress. The database used in this study comprises 50 healthy and 50 distressed fetal heart rate signals with severe intrauterine growth restriction. The Cascade analysis investigates six kernel based entropy measures on fetal heart rates discrimination, and compares them to four standard entropies. The study presents a statistical evaluation of the discrimination power of each parameter (paired t-test statistics and distribution spread). Simulation results showed that the distribution ranges in $80 \%$ of the entropy parameters in the distressed heart group are higher than those in the healthy control group. Moreover, the results show that it is advantageous to choose Circular entropy then Cauchy entropy $(p<0.001)$ over the standard techniques, in order to discriminate fetal heart rates.
\end{abstract}

Index Terms-Circular, Triangular, Exponential, Gaussian, Cauchy and Spherical Entropy Parameters, Alternative Entropy Parameters, Fetal Heart Rate, Discrimination, Statistical Signal Processing.

\section{INTRODUCTION}

Nonlinear and statistical analysis of Fetal Heart Rate (FHR) is crucial for evaluating the fetal condition. Despite the widespread use of electronic FHR monitoring, its effect on decreasing the risk of fetal mortality has not been fully established [1], [2], [3]. To reduce inter- and intra- observer variability of visual analysis, computerized analysis was developed, but it did not result in significant clinical improvement [2]. Accordingly, there have been many efforts to develop new monitoring methods to provide an automatic differentiation between healthy and distressed fetal conditions [4]. In 2006, the identification of risky conditions during pregnancy was thought to be open to univariate analysis, multivariate or to both to determine the potential of FHR in screening for distressed fetuses [5]. Later, Ferrario et al. considered mul- tiparametric approaches which improved the identification of risky conditions specifically in Fetal Heart Variability (FHV) [6]. Entropy quantification was used for such discrimination and for prediction purposes. Entropy analysis measures the correction and persistence of a signal in a nonlinear mathematical approach to quantify the irregularity or disorder and complexity of a system [7], [8], [9], [10], [11], [12], [13]. Ferrario et al have used both approximate entropy and sample entropy, Ferrario et al have also used ANOVA test [6]. In 2013, Liu et al. have used different entropy measures including the Approximate entropy (ApEn), Sample entropy (SamEn), fuzzy entropy and multi-scale Fuzzy entropy to discriminate between healthy fetuses and fetuses with heart failure [14]. Later, these methods have also been used by Lim et la. to discriminate between healthy, severe intrauterine growth restricted fetuses and non severe intrauterine growth restricted fetuses [4]. In 2015, Mekyska et al. used cutting edge kernel based entropy parameters (KBEPs) in speech signal processing [15], and Zaylaa et al. applied few of these parameters to Electroencephalogram (EEG) [10]. However, according to our literature research kernel-based entropy parameters were not applied on FHR discrimination.

As severe intrauterine growth restricted fetuses reveal distressed fetal heart rate, the most sensitive parameter (i.e. considered as a predicting parameter) to the change in the heart rate remains under study. For this purpose, it was essential for us to carry out an exploratory study on the nonlinear entropy parameters to detect the parameters that are utmost sensitive to heart rate changes. We aim to utilize different cutting edge kernel-based entropy parameters. Our database includes data from 50 healthy individuals and 50 individuals with distressed FHRs. The analysis is applied on healthy fetuses and severe intrauterine growth restricted fetuses (distressed fetuses) in the third trimester. To evaluate the results quantitatively, we make use of the paired t-test, and qualitatively we visualize the kernel entropy results using the box plots. To improve the classification algorithm of fetal heart rates and to predict fetus distress, it is essential to choose the best classifier [16]. However, classifiers require the optimization of feature selection.

The used methodology to extract features of FHR data is 


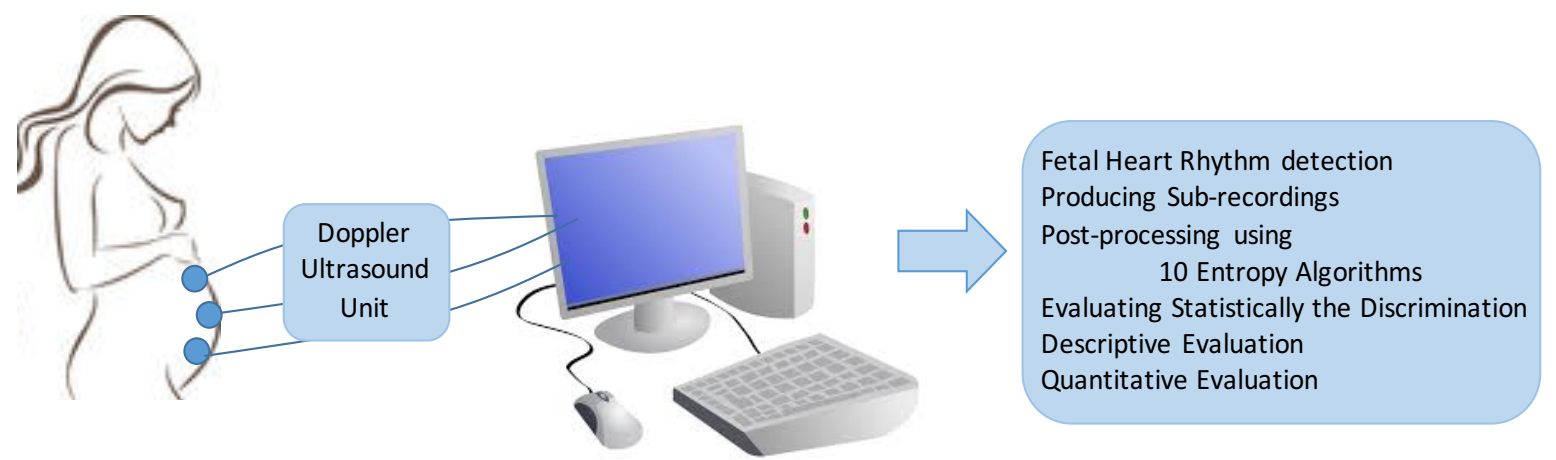

Fig. 1: The flowchart of FHR recording and complexity analysis through standard and kernel-based entropy.

analogous to the work of Mekyska et al. and our previous work Zaylaa et al. which was carried out on Electroencephalogram. The scenario is to use a cascade of nonlinear and statistical analysis. This includes investigating the performance of entropy measures on fetal heart rates, and comparing them with standard ApEn and SamEn as used by Liu et al, and the modified Shannon's entropy, i.e. Renyi entropy order (2) and (3). The second step is to use paired t-test analysis to compare the extracted entropy features between healthy and distressed subjects.

\section{Materials And Methods}

The major steps of this study are summarized in the flow chart in Fig. 1. Fig. 1 showcases the fetus, the electrodes, Doppler ultrasound unit/device and the entropy methods utilized to extract complexity parameters. The statistical evaluation used comprises the descriptive distribution plots and the quantitative paired t-test.

\section{A. Doppler Ultrasound Machine}

To collect FHR data, University of Tours and Altais Technology in Tours, France developed a pulse Doppler Ultrasound system, it is composed of four transducers and five gates. Single element transducers were placed on the maternal abdomen. These non-focused transducers are circular with a radius of $6.75 \mathrm{~mm}$. Ultrasound waves transmitted to the fetus were of sinusoidal pulses of $2.25 \mathrm{MHz}$ frequency with an acoustic power limited to $1 \mathrm{~mW} / \mathrm{cm}^{2}$. The signals backscattered by the fetal heart were acquired with a pulse repetition period of $1 \mathrm{~ms}$ and quantized over 12 bits. For each recording, forty demodulated signals (twenty signals in quadrature and twenty signals in phase) were then amplified to compensate for the attenuation of $1 \mathrm{~dB} / \mathrm{cm} / \mathrm{MHz}$. From the forty signals, the FHR was evaluated every $250 \mathrm{~ms}$, yielding 7200 samples issued every 30 minutes.

\section{B. Subjects and Protocol}

After having localized the fetal heart with the Doppler Ultrasound scanner, 100 Doppler recordings, each observed for 30 minutes were acquired at Bretonneau Hospital CHRU in Tours, France. The consent of each patient was obtained and the study was approved by the Ethics Committee of the Clinical Investigation Center for Innovative Technology of Tours (CIC-IT 806 CHRU of Tours). All patients were over eighteen years of age and pregnancies were single. The data set comprised 50 healthy and 50 distressed fetus recordings. For this clinical protocol, the gestational ages of fetuses ranged from 25 to 39 weeks. Distressed fetuses were identified with severe Intra-Uterine Growth Restricted (IUGR).

Two FHR data sets were used from the recorded database. Two distinct phases were distinguished in the third trimester of pregnancy as healthy fetus (H-FHR) or distressed fetus (D-FHR). Fig. 2 illustrates two FHR signals recorded for 3 minutes. The electrodes were introduced on the abdomen of the pregnant mothers who were in the third trimester of pregnancy. Pregnancies were either normal and ready for vaginal labor, or abnormal pregnancies where the fetus is in distress due to intrauterine growth restriction and should be subject to cesarean labor.

\section{Pre- and Post-Processing}

A pre-processing algorithm was applied as described in previously reported studies [5], to remove artifacts caused by fetal and maternal movements.

After preparing FHR recordings and before commencing nonlinear entropy analyses, the signals were post-processed. For each recording, a window size of 250 and a shift size of 25 were applied to produce sub-signals.

\section{Nonlinear Entropy Methods}

The nonlinear analysis was applied on the sub-signals produced in the post-processing step. Entropy measures help in analyzing these signals by providing the amount of information they carry (Renyi entropy) or by estimating the system's complexity upon computing them (ApEn and SamEn and others).

1) Renyi Entropy: It is a mathematical generalization of Shannon's Entropy, it preserves the addition of statistically independent systems, and compatible with Kolmogorov's probability axioms [15]. The Renyi entropy of order $\alpha$, where $\alpha \geq 0$ and $\alpha \neq 1$ is defined as: 


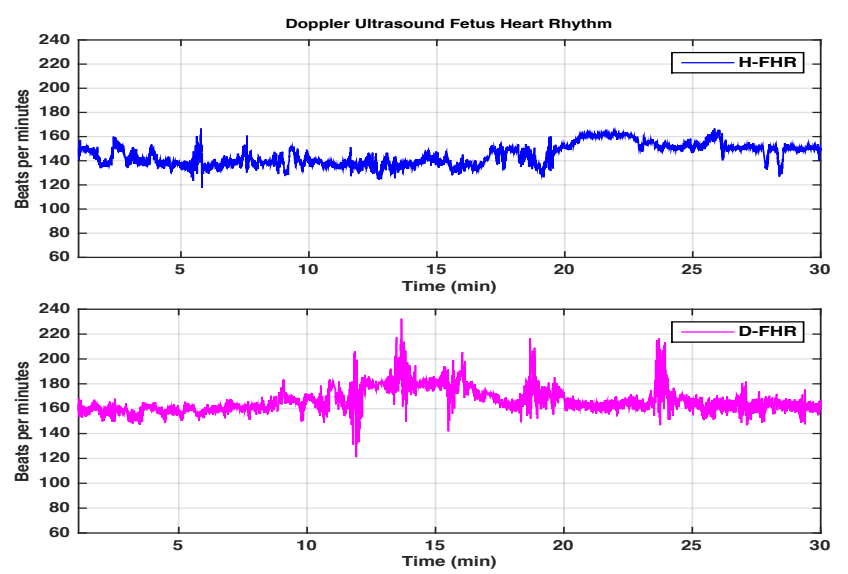

Fig. 2: Fetal Heart Rate (FHR) recordings issued from different fetuses for 30 minutes at Bretonneau Hospital in Tours, France. (a) A healthy FHR recording and (b) a distressed FHR recording.

$$
H_{\alpha}=\frac{1}{1-\alpha} \log \left(\sum_{i=(0)}^{n} P_{i}^{\alpha}\right)
$$

where $X$ is a discrete random variable with outcomes $1,2, \ldots \ldots, n$ and corresponding probabilities $P_{i}=\operatorname{Pr}(X=i)$ and $\log$ is based 2. Renyi entropy has unique properties depending on the data spread and how the probability changes as the order increases. Therefore, it is beneficial to study Renyi entropy of order 2, 3 etc.

2) Sample Entropy: measures the complexity without including self similar patterns.

$$
\operatorname{SamEn}(m . r)=\left[\phi^{m}(r)-\phi^{m+1}(r)\right],
$$

where $\phi^{m}(r)$ is given by:

$$
\phi^{m}(r)=\log \left(\frac{1}{N-m} \sum_{i=1}^{N-m} C_{i}^{m}(r)\right)
$$

and $C_{i}^{m}$ :

$$
C_{i}^{m}=\frac{1}{N-m+1} \sum_{j=0, i \neq j}^{N-m+1} \Theta(i, j, r),
$$

where $\Theta$ is the Heaviside function. The algorithm builds up a number of runs of points matching within the tolerance $r$ until there is no match, and keeps track of template matches continued until the end of the data [16].

3) Kernel-Based Entropy: is a method extensively used to assess the predictability and regularity of changes in a time series [15]. For a given time series of entries $s(1), s(2), s(3), \ldots \ldots, s(N)$ that are equally spaced in time, we define the embedding dimension as the length of compared runs and denote it as $m$, and a filtering variable, $r$, which is acts as the embedding tolerance. Furthermore a sequence of vectors, $X(i)$ is defined as:

$$
\mathbf{X}_{i}=[s(i), s(i+1), \ldots, s(i+m-1)]
$$

and for each $i, 1 \leq i \leq N-m+1$, we define the center of pressure position angle, $\phi^{m}(\mathrm{r})$, as:

$$
\phi^{m}(r)=\frac{1}{N-m+1} \sum_{i=1}^{N-m+1} \ln C_{i}^{m}(r)
$$

and we define $C_{i}^{m}$ as in Eq. 4 such that $\mathrm{i}$ could be equal to $\mathrm{j}$, $\Theta(i, j, r)$ is replaced by $k(i, j, r)$ and the kernel-based entropy is given as:

$$
\text { Kernel Based-En(m.r) }=\left[\phi^{m}(r)-\phi^{m+1}(r)\right] .
$$

$\mathrm{k}(\mathrm{i}, \mathrm{j}, \mathrm{r})$ in Eq. 6 is the kernel, when this kernel describes a Heaviside function $\Theta$ then the resulting entropy value is the Approximate Entropy (ApEn). Moreover, the kernel can be changed to the following kernels [17]:

Triangular Kernel:

$$
\mathbf{k}(i, j, r)=1-\frac{\left\|\mathbf{X}_{i}-\mathbf{X}_{j}\right\|}{r},
$$

the corresponding entropy is called Triangular entropy and denoted through this work by TriEn.

Spherical Kernel:

$$
\mathbf{k}(i, j, r)=1-\frac{3}{2} \frac{\left\|\mathbf{X}_{i}-\mathbf{X}_{j}\right\|}{r}+\frac{1}{2}\left(\frac{\left\|\mathbf{X}_{i}-\mathbf{X}_{j}\right\|}{r}\right)^{3},
$$

the corresponding entropy is called Spherical Entropy and denoted by SphEn.

Cauchy Kernel:

$$
\mathbf{k}(i, j, r)=\frac{1}{1+\frac{\left(\left\|\mathbf{X}_{i}-\mathbf{X}_{j}\right\|\right)^{2}}{r}},
$$

the corresponding entropy is called Cauchy Entropy and denoted by CaEn.

Circular Kernel:

$\mathbf{k}(i, j, r)=\left\{\begin{array}{c}\left(\frac{2}{\pi} \arccos \frac{\left(\left\|\mathbf{X}_{i}-\mathbf{X}_{j}\right\|\right)}{r}-\frac{2}{\pi} \frac{\left(\left\|\mathbf{X}_{i}-\mathbf{X}_{j}\right\|\right)}{r}\right) \\ \left(\times \sqrt{\left(1-\frac{\left(\left\|\mathbf{X}_{i}-\mathbf{X}_{j}\right\|\right)^{2}}{r}\right.}\right),\end{array}\right.$

the corresponding entropy is called Circular Entropy and denoted by CirEn.

Exponential Kernel:

$$
\mathbf{k}(i, j, r)=\frac{1}{2 r^{2}} \times \exp \left(-\left\|\mathbf{X}_{i}-\mathbf{X}_{j}\right\|\right)^{2}
$$

the corresponding entropy is called Exponential Entropy and denoted by ExpEn.

Gaussian Kernel:

$$
\mathbf{k}(i, j, r)=\frac{1}{10 r^{2}} \times \exp \left(-\left\|\mathbf{X}_{i}-\mathbf{X}_{j}\right\|\right)^{2} .
$$

the corresponding entropy is called Gaussian Entropy and denoted by GauEn.

\section{Statistical Evaluation of Entropy Parameters}

StatVIEW software was used to perform paired t-test and the significant level was set to 0.05 Bonferroni corrected by 


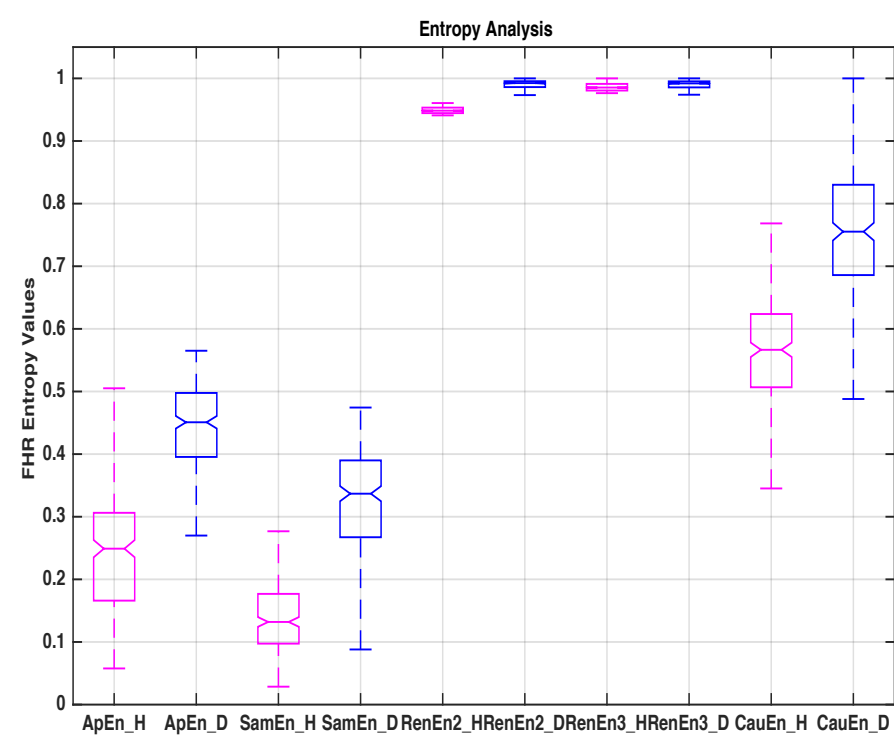

(a)

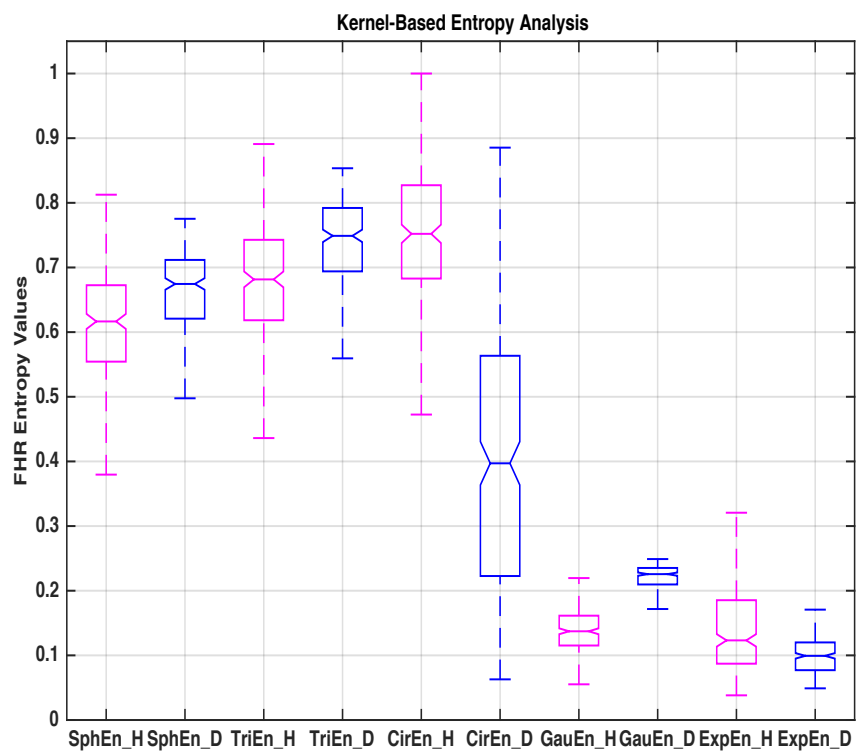

(b)

Fig. 3: Box plots of the Nonlinear entropy analysis between healthy fetuses (vaginal delivery) coded in magenta and distressed fetuses (fetuses undergoing emergency cesarean delivery) coded in blue. (a) The Approximate, Sample, Renyi order 2, Renyi order 3 and Cauchy's entropies, ApEn, SamEn, RenEn1, ReniEn2 and CauEn, respectively. (b) The Spherical, Triangular, Circular, Gaussian and Exponential entropies, SphEn, TriEn, CirEn1, GauEn and ExpEn, respectively.

a factor 10 due to the fact that 10 comparisons are done for the 10 entropy parameters $(\sigma=0.005$ instead of 0.05$)$.

\section{RESUlts}

To increase the sensitivity of this exploratory study, the entropy analysis parameters have been applied on each subsignal produced after windowing the FHR recording.

\section{A. Qualitative Evaluation}

To test the discrimination between healthy and distressed FHRs, Fig. 3 sets out the box plots of the nonlinear entropy measures between healthy fetuses, i.e. ready for vaginal delivery, coded in magenta and distressed fetuses, i.e. intrauterine growth restricted fetuses which need emergency cesarean delivery, coded in blue. (a) Approximate, Sample, Renyi order 2, Renyi order 3 and Cauchy's entropies, ApEn, SamEn, RenEn1, ReniEn2 and CauEn, respectively. (b) Spherical, Triangular, Circular, Gaussian and Exponential entropies, SphEn, TriEn, CirEn, GauEn and ExpEn, respectively.

The box plot represents the distribution of entropy methods of healthy and distressed FHRs based on the five number summary: minimum, first quartile, median, third quartile, and maximum. The central rectangle of the box plot spans the first quartile to the third quartile (the interquartile range or IQR which is the likely range of variation).

The four standard Entropies are Approximate, Sample, Renyi order 2 and Renyi order 3, denoted ApEn, SamEn, RenEn1 and ReniEn2, respectively. While the kernel-based entropies are Cauchy, Spherical, Triangular, Circular, Gaussian and Exponential entropies, denoted CauEN, SphEn, TriEn, CirEn1, GauEn and ExpEn, respectively.

\section{B. Quantitative Evaluation}

The mean difference (MD) measure of irregularity, which corresponds to the average value obtained for all sub-signals extracted from the 30 minutes original data, has been computed. Absolute MD measures the absolute separation between the two different FHR data sets and the results are reported in Table I.

Note that for statistical significance the p-value should be $<0.05 / 10=0.005$, since we did 10 paired t-test comparisons (this is known as Benforroni correction for multiple comparisons). The p-value in all the tests is $<0.001$, thereby reveals that the discrimination was significant.

\section{Discussion AND CONCLUSION}

It was noted that the distribution ranges of 8 out of 10 entropy parameters (ApEn, SamEn, RenEn2, RenEn3, CauEn, SphEn, TriEn and GauEn) in the distressed heart group are larger than those in the healthy control group. The reason behind this could be due to the fatal arrhythmias which accompanies the course of the heart failure, such as supraventricular ectopic beats, ventricular ectopic beats and even, ventricular or atrial fibrillation. This can cause the acute fluctuations in the FHR. In addition, the entropy values of FHR recordings of the distressed fetuses without arrhythmia, 
TABLE I: Statistical evaluation of the nonlinear entropy analysis using paired t-test between healthy fetuses (vaginal delivery) entropy, and distressed fetuses (fetuses diagnosed for emergency cesarean delivery) entropy after Bonferroni-correction.

\begin{tabular}{|c|c|c|c|}
\hline Entropy Technique & Absolute Mean Difference & Absolute t-Value & P-Value \\
\hline $\operatorname{ApEn}_{H}, \operatorname{ApEn}_{D}$ & 0.193 & 22.257 & $<0.0001$ \\
\hline $\operatorname{SamEn}_{H}, \operatorname{SamEn}_{D}$ & 0.180 & 25.255 & $<0.0001$ \\
\hline $\operatorname{RenEn} 2_{H}, \operatorname{RenEn} 2_{D}$ & 0.042 & 73.783 & $<0.0001$ \\
\hline $\operatorname{RenEn} 3_{H}, \operatorname{RenEn} 3_{D}$ & 0.004 & 6.528 & $<0.0001$ \\
\hline $\operatorname{CauEn}_{H}, \operatorname{CauEn}_{D}$ & 0.318 & 22.462 & $<0.0001$ \\
\hline SphEn $_{H}$, SphEn $_{D}$ & 0.062 & 7.793 & $<0.0001$ \\
\hline $\operatorname{TriEn}_{H}$, TriEn $D$ & 0.071 & 8.331 & $<0.0001$ \\
\hline $\operatorname{CirEn}_{H}, \operatorname{CirEn}_{D}$ & 0.380 & 17.362 & $<0.0001$ \\
\hline $\operatorname{ExpEn}_{H}, \operatorname{ExpEn}_{D}$ & 0.081 & 30.016 & $<0.0001$ \\
\hline $\mathrm{GauEn}_{H}, \mathrm{GauEn}_{D}$ & 0.038 & 8.706 & $<0.0001$ \\
\hline
\end{tabular}

as could be the case in the 2 out of 10 entropy parameters, have a regular change caused by the weakening of the regulatory functions of the autonomic nervous system. Thereby, the mean entropy values seemed to be more regular for the distressed group as compared to the healthy group (CirEn and ExpEn) (see Fig. 3).

Note that as the fetal activity increases the disorder of non-similar patterns increase, accordingly the disorder of self-similar patterns decreases while the disorder of non-self similar patterns increases [18]. This plays a role of a criteria for classifying the pace of the activity of the fetus whether it is increasing or decreasing. Before it was thought that as the fetal activity increases, the disorder of all patterns including self-similar patterns decreases.

The findings derived from the normalized values showcased in Fig. 3 and table I suggest that the best parameters that provide the distinction between healthy and distressed fetuses is the circular entropy CirEn and Cauchy entropy CauEn (with an absolute mean difference $M D=0.380$ and 0.318 , respectively). Therefore, it was advantageous to choose circular entropy, CirEn, and Cauchy entropy, CauEn, with $p<0.001$ over the standard ApEn, SamEn and even the modified Shannon's entropy of order 2 and 3. Two Kernelbased entropy parameters surpassed the standard ApEn and the measured alternatives. Research results also showed that the weaker parameter is RenEn3.

Table I showcases that the maximum absolute t-values corresponds to RenEn2 (73.783) followed by ExpEn (30.016) Therefore, the choice of the feature is dependent on the application at hand.

Note that the paired t-test statistics was utilized after applying the Kolmogorov-Smirov test in each entropy method and on each parameter, for both healthy and distressed FHR groups. This test showed that most variables are either normally distributed $(p>0.05)$ or marginally normally distributed.

But as the distribution profiles are not only normal, further statistical tests have to be utilized too. Besides, other entropy features can be studied and compared with the most powerful parameters resulting from this exploratory study. Moreover, the extracted features (entropy parameters), being a part of an automated analysis procedure to improve fetal heart rate monitoring and discrimination, need to be tested in a variety of classifiers.

\section{ACKNOWLEDGEMENTS}

The authors gratefully acknowledge Dr. Jean-Marc Girault and Prof. F. Perrotin and his team in the Obstetric Department at CHRU-Bretonneau Hospital of Tours for the Fetal Heart Rate signals. The authors would like also to thank Miss Faten I. Khatib from Sharp Chula Vista Medical Center for editing the paper.

\section{REFERENCES}

[1] A. P. Bulletin, "Intrapartum fetal heart rate monitoring: nomenclature, interpretation, and general management principles," Obstet Gynecol, vol. 114, no. 106, pp. 192-202, 2009.

[2] A. Zaylaa, J. Charara, and J. M. Girault, "Reducing sojourn points from recurrence plots to improve transition detection: Application to fetal heart rate transitions," Computers In Biology and Medicine, september 2014.

[3] A. Zaylaa, J.-M. Girault, and J. Charara, "Unbiased recurrence plot quantification of chaotic dynamic systems by eliminating sojourn points," in Proc. ICABME. IEEE, 2013, pp. 187-190.

[4] J. Lim, J. Y. Kwon, J. Song, H. Choi, J. C. Shin, and I. Y. Park, "Quantitative comparison of entropy analysis of fetal heart rate variability related to the different stages of labor," Early human development, vol. 90, no. 2, pp. 81-85, 2014.

[5] D. Rouvre, "Caracterisation de l'activite foetale: mise en oeuvre d'un dispositif d'enredistrement et analyse des signaux doppler multidimensionnels," Ph.D. dissertation, Universite Francois Rabelais de Tours, Tours, France, 2006.

[6] M. Ferrario, M. G. Signorini, and G. Magenes, "Complexity analysis of the fetal heart rate variability: early identification of severe intrauterine growth-restricted fetuses," Medical \& biological engineering \& computing, vol. 47, no. 9, pp. 911-919, 2009.

[7] S. Oudjemia, A. Zaylaa, J. Charara, and J.-M. Girault, "Delta-fuzzy similarity entropy to discriminate healthy from sick fetus," in Proc. ICABME. IEEE, 2013, pp. 1-4. 
[8] A. Zaylaa, "Analysis and extraction of complexity parameters of biomedical signals," Ph.D. dissertation, François-Rabelais University of Tours, 2014.

[9] A. Zaylaa, S. Oudjemia, J. Charara, and J.-M. Girault, "n-order and maximum fuzzy similarity entropy for discrimination of signals of different complexity: Application to fetal heart rate signals," Computers in Biology and Medicine, March 2015.

[10] A. J. Zaylaa, A. Harb, F. I. Khatib, Z. Nahas, and F. N. Karameh, "Entropy complexity analysis of electroencephalographic signals during pre-ictal, seizure and post-ictal brain events," in Proc. ICABME. IEEE, 2015, pp. 134-137.

[11] A. J. Zaylaa, J. Charara, and J. M. Girault, "Advanced discrimination between healthy and intrauterine growth restricted fetuses by unbiased recurrence plots," Advanced Techniques in Biology and Medicine, 2016, in press.

[12] K. Al Ali, M. Doumit, M. Atoui, A. Zaylaa, F. Karameh, and Z. Nahas, "Seizure initiation with focal electrically administered seizure therapy (feast)," Neuropsychopharmacology, vol. 40, no. S1, pp. S443-S611, 2015.

[13] T. Akiki, M. Doumit, A. Zaylaa, F. Karameh, and Z. Nahas, "Contrasting non-linear dynamic analyses of eeg in alert and sedated states," Neuropsychopharmacology, vol. 40, no. S1, pp. S106-S271, 2015.

[14] C. Liu, K. Li, L. Zhao, F. Liu, D. Zheng, C. Liu, and S. Liu, "Analysis of heart rate variability using fuzzy measure entropy," Computers in biology and Medicine, vol. 43, no. 2, pp. 100-108, 2013.

[15] J. Mekyska, E. Janousova, P. Gomez-Vilda, Z. Smekal, I. Rektorova, I. Eliasova, M. Kostalova, M. Mrackova, J. B. Alonso-Hernandez, M. Faundez-Zanuy, and k. Lopez-de Ipina, "Robust and complex approach of pathological speech signal analysis," Neurocomputing,, 2015.

[16] L. Chen, Y. Zhao, J. Zhang, and J.-Z. Zou, "Automatic detection of alertness/drowsiness from physiological signals using wavelet-based nonlinear features and machine learning," Expert Systems with Applications, 2015.

[17] K. Adjerid, P. Fino, M. Habib, A. Rezaei, S. Ross, and T. Lockhart, "Comparing postural stability entropy analyses to differentiate fallers and non-fallers," Annals of biomedical engineering, 2014.

[18] H. Gonçalves, J. Bernardes, A. P. Rocha, and D. Ayres-de Campos, "Linear and nonlinear analysis of heart rate patterns associated with fetal behavioral states in the antepartum period," Early human development, vol. 83, no. 9, pp. 585-591, 2007. 